\title{
A COMPARISON OF TWO INTERACTION MATRIX CODING TECHNIQUES USED IN A GIS-BASED TOOL FOR AIR QUALITY ASSESSMENT
}

\author{
M. MAVROULIDOU ${ }^{1, *}$ \\ S.J. HUGHES ${ }^{2}$ \\ E.E. HELLAWELL ${ }^{2}$
}

\author{
${ }^{1}$ Department of Engineering Systems \\ London South Bank University \\ 103 Borough Road, London, SE1 OAA, U.K. \\ ${ }^{2}$ School of Engineering \\ Department of Civil Engineering \\ University of Surrey, Guildford, Surrey, GU2 7XH, U.K. \\ *to whom all correspondence should be addressed: \\ e-mail: mavroum@Isbu.ac.uk
}

Received: 31/03/06

Accepted: 30/05/06

\begin{abstract}
The paper describes the development of a fast and easy-to-use qualitative tool for preliminary assessments of urban air quality related to road traffic. The tool is particularly aimed at the ability and budget of local government. It uses a novel interaction matrix-type methodology combined with mapping overlay, performed via a GIS. More specifically, the interaction matrix provides the weighting factors, which show the impact of each variable involved in a system on the target variable, air quality, as well as on the system as a whole. These weighting factors are used in the GIS to produce vulnerability maps. The maps visualise vulnerability to air pollution due to the combined effect of a number of interacting factors, and thus indicate areas susceptible to poor air quality. This results in a considerable reduction in computing time and complexity compared to the use of a sophisticated numerical model, as the user of the GIS tool only needs to perform mapping overlays in the GIS (using the previously derived weighting factors).

The particular aim of this study was to compare two different methods for quantifying the interactions between variables in the matrix. The first method used constant coefficients, whose values are based on parametric studies performed using an advanced dispersion model or on good engineering judgement. The second method used a more sophisticated and versatile quantification of the interactions between variables, via analytical or semi-empirical relationships. In the latter method, the matrix was formulated computationally, so that the interaction weightings for different conditions can be obtained automatically.

The technique was applied to the case study of an urban area with a high traffic throughput, in the UK. Two different interaction matrices were constructed for urban air quality linked to road traffic, based on the above methods. The GIS results based on both matrix methodologies were compared to the results of a more intensive dispersion numerical model in terms of pollutant dispersion patterns and hot spots. Both sets of results were shown to compare favourably with those of the numerical model. The results based on the more sophisticated matrix coding were found to be in closer agreement with those of the numerical model.
\end{abstract}

KEYWORDS: Air quality management, interaction matrix, Geographical Information System

\section{INTRODUCTION}

Recent changes in the UK legislative requirements for air quality standards, specify that each local authority in the UK is obliged by law to assess air pollution levels within their administrative boundaries [1]. This is usually achieved via field measurements and numerical modelling. Sophisticated numerical models are normally expensive to acquire and may require specialised users, who are often beyond the budget of small-scale projects for local authorities. Moreover, they may require a large amount of detailed and accurate input data. 
Instrumentation to obtain such data is complex and costly and the necessary monitoring time can be lengthy. To save costs and time, local authorities need alternative tools for preliminary air quality assessments to identify problem areas requiring a more rigorous investigation, and exclude areas of low vulnerability from more detailed study.

The aim of this research, sponsored by the Engineering and Physical Sciences Research Council (EPSRC), was to develop such a qualitative tool that would be applied to assess current or future vulnerability to air pollution due to traffic, in the county of Surrey, UK. In Surrey, traffic levels and car ownership are amongst the highest in the country. This results in significant traffic congestion and hence, potentially high air pollution levels. The tool combines two techniques used independently in the field of environmental impact assessment. These are an interaction matrix and mapping overlay through a GIS. An overview of the methodology is shown in Figure 1 . The following section provides a more detailed presentation of the methodology.

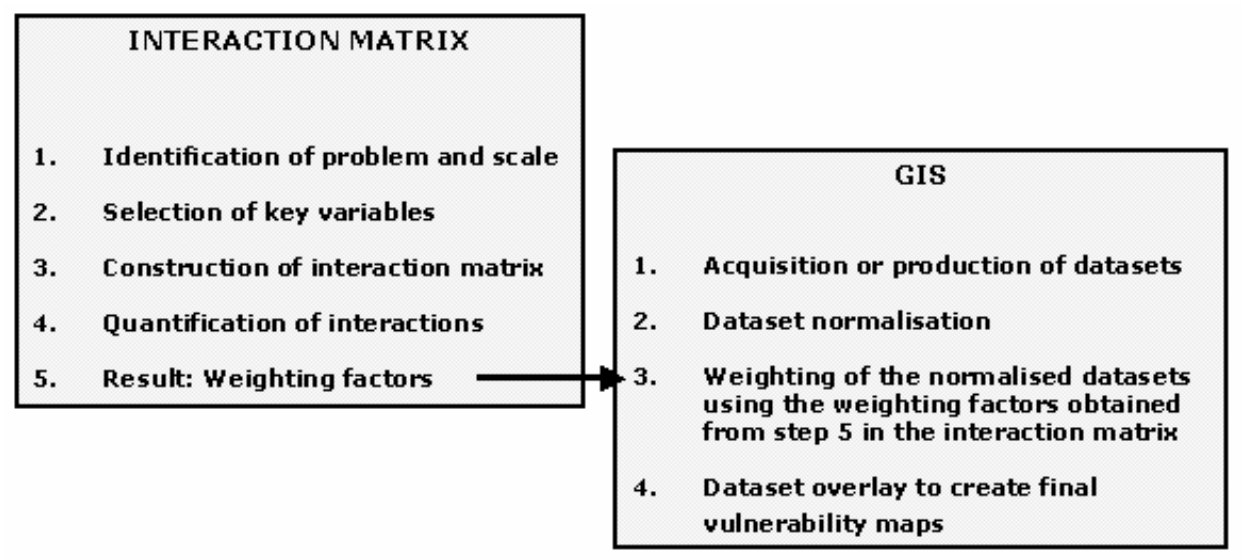

Figure 1. Overview of the methodology

\section{METHODOLOGY}

The interaction matrix methodology used in this paper was initially developed for rock engineering systems [2]. Unlike conventional interaction matrices [3], this methodology incorporates system analysis theory concepts and accounts for the relative importance of each variable in a system. It can therefore investigate the interactions of each element with other individual elements of the system as well as the interaction of each element of the system with the system as a whole.

The first stage in the development of the method consists in the selection of the key variables of the system being analysed. In this study six variables were identified (emissions from traffic, wind, atmospheric stability, surface roughness, buildings and air quality) and placed in the leading diagonal of a $6 \times 6$ matrix. The matrix thus obtained (see Table 1) involves 30 possible interactions between the variables. The variable constituting the focus of the study (i.e. air quality) was set as the last (i.e. sixth) element of the leading diagonal. Air quality was accounted for with reference to ground-level concentrations of the primary pollutant carbon monoxide, $\mathrm{CO}$ and assuming a static urban system. The effect of buildings on air quality was considered for the case of street canyons only. Outside the street canyon it was assumed that the variable 'buildings' was not relevant and a 5x5 matrix was used, as for open roads.

The difficulty arises in quantifying the interactions. Hudson [2] suggests four methods of different levels of complexity. The simplest and crudest approach is to use a binary quantification system. The interaction is either considered to be switched on (interaction value $=1$ ) or off (interaction value $=0$ ). A three-class system can be used to distinguish between negative (bad) and positive (good) effects ( -1 and +1 signs respectively). An extension of the binary coding is the expert rating, using a number of classes according to the intensity of the interaction. Hudson [2] suggests five classes, ranging from 0 (no interaction) to 4 (critical interaction). $A$ third approach is to use the slope of the $V_{i} v s . V_{j}$ curve to quantify the 
interactions (where $V_{i}, V_{j}$ are the system variables). At the highest level of sophistication, complete mathematical analysis could be performed.

In this study two different methodologies for the quantification of the interactions were implemented and assessed comparatively: a matrix based on constant interaction coefficients and another with varying interaction coefficients. The implementation of the two different matrix codings is explained below.

\subsection{Constant coefficient matrix}

The first interaction matrix coding used constant interaction coefficients, with Hudson's scaling convention of 0-4. However, in order to reduce subjectivity in the quantification, the interactions with respect to the target variable (i.e. column 6 in Table 1) were quantified using the advanced dispersion model ADMS-Urban (rather than expert rating). This was achieved through parametric studies in which each variable was incremented over a fixed range of values representative of Surrey towns. The interaction coefficients corresponded to the maximum possible effect of each variable on air quality. The remaining interactions were assigned values based on good engineering judgment as it was not possible to apply the dispersion model for their quantification. A detailed description of the methodology for air quality, applied to a small study area is given in [4].

Table 1. Interaction matrix for urban air quality in Surrey (constant coefficients)

\begin{tabular}{|l|l|l|l|l|l|l|l|}
\hline \multicolumn{7}{|c|}{} & $I_{i}$ \\
\hline \multicolumn{1}{|c|}{ Emissions from Traffic } & $I_{12}=0$ & $I_{13}=0$ & $I_{14}=0$ & $I_{15}=0$ & $I_{16}=4$ & $\mathbf{4}$ \\
\hline$I_{21}=1$ & Wind & $I_{23}=2$ & $I_{24}=0$ & $I_{25}=2$ & $I_{26}=4$ & $\mathbf{9}$ \\
\hline$I_{31}=0$ & $I_{32}=4$ & Stability & $I_{34}=0$ & $I_{35}=0$ & $I_{36}=2$ & $\mathbf{6}$ \\
\hline$I_{41}=0$ & $I_{42}=2$ & $I_{43}=2$ & Roughness & $I_{45}=1$ & $I_{46}=3$ & $\mathbf{8}$ \\
\hline$I_{51}=0$ & $I_{52}=2$ & $I_{53}=1$ & $I_{54}=4$ & Buildings & $I_{56}=3$ & $\mathbf{1 0}$ \\
\cline { 2 - 8 } & & & & & & Air Quality & $\mathbf{0}$ \\
\hline$I_{61}=0$ & $I_{62}=0$ & $I_{63}=0$ & $I_{64}=0$ & $I_{65}=0$ & & \\
\hline$Z_{j}$ & $\mathbf{1}$ & $\mathbf{8}$ & $\mathbf{5}$ & $\mathbf{4}$ & $\mathbf{3}$ & $\mathbf{1 6}$ & $\mathbf{3 7}$ \\
\hline
\end{tabular}

\subsection{Varying coefficient matrix}

The second method used a more sophisticated coding with varying interaction coefficients. These corresponded to the slope of each curve $V_{i}$ vs. $V_{j}$ (i.e. the third matrix coding suggested by Hudson [2]). However, to address non-linearity in the relationships, an incremental methodology was used in this study: different matrices with different coefficients were constructed for each step variation of the system variables. Thus, the matrix coefficients represented the change in the values of each variable for a step change in the values of the other variables (changing one at a time). The resulting changes were normalised with respect to the maximum range of each variable for the given location. The slope of the binary relationships between each pair of variables was then evaluated. This gave the final values of each interaction.

As the values of interactions are also a function of the distance from the pollution source, this incremental process was repeated for different increments of distance. This generated different sets of matrices for each distance from the source. The whole process, described in detail in [5], was automated using a specifically designed Java computer program.

For the relationships between variables, the following expressions were used:

a) For interactions related to the variable 'air quality' (interactions $I_{16}, I_{26}, I_{36}, I_{46}$ ), a Gaussian line source equation was used, namely:

$$
C(x, z)=\sqrt{\frac{2}{\pi}} \frac{Q}{U \sigma_{z}} \exp \left[-\frac{z^{2}}{2 \sigma_{z}^{2}}\right]
$$


where $C(x, z)$ is the pollutant concentration with dimensions of mass per unit volume $(M$ $\mathrm{L}^{-3}$ ), $Q$ is the emission rate (i.e. pollutant mass per unit length, $\mathrm{M} \mathrm{L}^{-1} \mathrm{~T}^{-1}$ ), $\mathrm{U}$ is the wind speed at emission level $\left(L T^{-1}\right), z$ is the receptor height $(L)$ and $\sigma_{z}$ the vertical dispersion parameter (with units of length, L). Note that Eqn (1) is written for a wind direction normal to the road axis. This was considered appropriate, as the roads in an urban network are at random directions with respect to the that of the wind.

The dispersion coefficients were calculated from Pasquill-Briggs relationships [6] for urban and rural conditions respectively and an additional term $\sigma_{\mathrm{zt}}=2 \mathrm{~m}$ to account for initial dilution due to car-induced turbulence $[7,8]$.

For open roads, the wind speed $U\left(z_{e}\right)$ at emission level (assumed to be $2 \mathrm{~m}$ ) was calculated by a power law relationship [9], as:

$$
\mathrm{U}\left(\mathrm{Z}_{\mathrm{e}}\right)=\mathrm{U}_{10}\left(\frac{\mathrm{Z}_{\mathrm{e}}}{10}\right)^{\mathrm{p}}
$$

where, $\mathrm{Z}_{\mathrm{e}}$ is the emission level, $\mathrm{U}\left(\mathrm{z}_{\mathrm{e}}\right)$ and $\mathrm{U}_{10}$ are the wind speeds at emission level and at $10 \mathrm{~m}$ respectively. The parameter $\mathrm{p}$ varies as a function of atmospheric stability and roughness length according to relationships provided by Irwin [9].

The same relationship was used to quantify the effects of stability and roughness on the wind speed (interactions $I_{32}$ and $I_{42}$ respectively).

b) The effects of the wind and roughness on atmospheric stability (interactions $I_{23}$ and $I_{43}$ respectively) were calculated based on modified Pasquill's tables, which included stability class $\mathrm{G}$ and assumed intermediate stability sub-classes [10].

c) For the effects of buildings on air quality (interaction $I_{15}$ ), a simplified version of the OSPM model was used [8].

d) For the effect of the buildings on the wind speed (interaction $\mathrm{I}_{52}$ ), a logarithmic reduction of the wind speed at roof level, towards the bottom of the street, was assumed [8].

$U_{b}=0.8 U_{\text {roof }}\left(\frac{\log \left(h_{0} / z_{0}\right)}{\log \left(H_{b} / z_{0}\right)}\right)^{p}$

where $U_{b}, U_{\text {roof }}$ are the wind speeds at street and roof level respectively; $h_{0}$ is the initial (immediate) dispersion in the wake of the vehicles and $\mathrm{H}_{\mathrm{b}}$ is the average height of the street canyon buildings. Parameter $p$ varies as a function of atmospheric stability and roughness length $z_{0}$, as described above [9].

e) The effect of the wind speed on emissions was assumed to be zero for wind speeds within the ranges of pedestrian comfort and/or safety, and non-zero (values based on engineering judgment) above these ranges, when car use is expected to be higher or when problems in circulation may occur due to windstorm effects.

f) The remaining interactions were set to zero for the scale and area of study.

\subsection{Weighting factors}

For each of the matrix techniques (constant or varying coefficients), two separate sets of weightings were obtained:

a) the specific weightings, which show the effect of all other variables on the target variable.

These correspond to the interaction coefficients in the column containing the target variable (column 6 in Table 1). Negative and positive signs were used to distinguish between positive and negative effects on air quality respectively.

b) the overall weightings, showing the impact of each variable on the system as well as the effect of the system on each variable. The total overall weighting of each variable is obtained by summing (in terms of absolute values) the individual row and column 
containing that variable (see Table 1 , final column $\Sigma \mathrm{l}_{\mathrm{j}}$ and final row, $\Sigma \mathrm{l}_{\mathrm{i}}$ respectively) and expressing this sum as a percentage of the total sum of all interaction values.

\subsection{Geographic Information System}

The weighting factors obtained from the interaction matrices, were imported into the GIS to create vulnerability maps. The location of the study area in this paper, the Borough of Guildford, in Surrey, UK, is shown in Figure 2. Many roads within the area are major commuter routes with high traffic throughput, typically with average daily flows twice the UK National average [11]. The modelled area comprised Guildford town centre (an area of urban roughness characteristics) and the surrounding area/villages (of suburban to rural roughness characteristics).

Initially, spatial datasets of the selected key variables were obtained for the study area. The traffic volumes and speeds on the modelled road network were obtained from the Surrey County Transportation Model, CTM95 [12]. The peak hour CO emissions were estimated according to the Design Manual for Roads and Bridges [13], which uses the UK Transport Research Laboratory (TRL) database, the COPERT II computer program and the Swiss/German Workbook on emission factors for road vehicles. Each spatial dataset was normalised with respect to minimum and maximum values. The normalised datasets were multiplied by the respective weighting factor for each variable (obtained from the interaction matrix) and overlaid, to generate vulnerability maps identifying pollution sensitive zones.

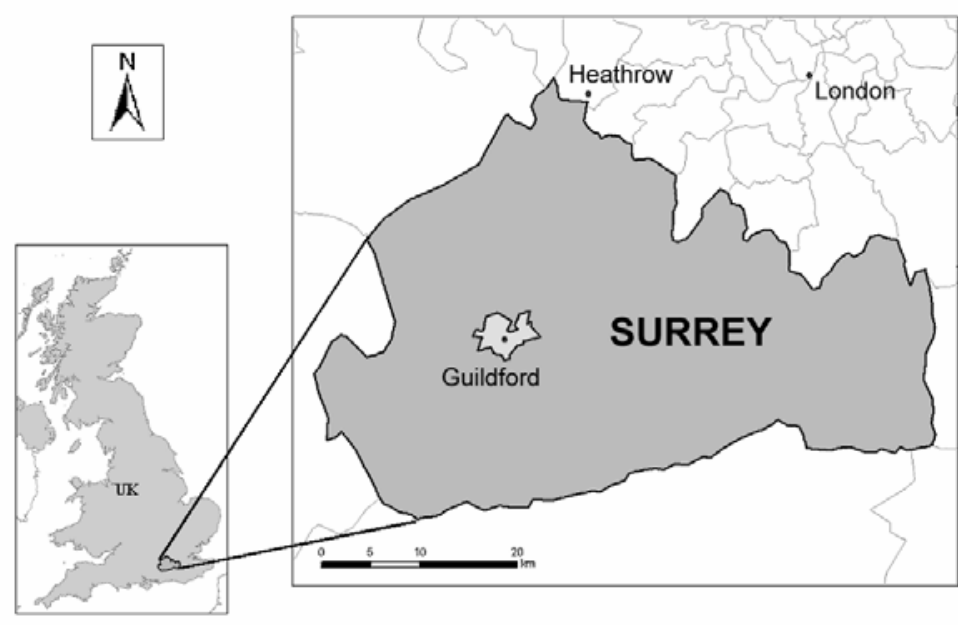

Figure 2. Study area: the county of Surrey and the wider urban area of Guildford

\section{RESULTS AND DISCUSSION}

Figures 3 and 4 show examples of vulnerability maps for current traffic levels in the wider borough of Guildford in Surrey, produced by this tool. They correspond to the constant and varying coefficient matrix methodologies respectively. The maps depict the vulnerability zones numbered in ascending order (1-12) from the lowest to highest air pollutant concentrations. They are based on a "worst-case scenario", which corresponds to the maximum possible CO ground level concentrations for ranges of values of each variable representative of those encountered in the study area. These maps were compared (in terms of patterns showing sensitivity to air pollution) to those from the advanced dispersion model ADMS-Urban (Figure 5).

Figure 5 also shows $\mathrm{NO}_{2}$ tube measurements for the area and year of study. These were included for indicative purposes only, due to the lack of real time measurements of $\mathrm{CO}$ for the wider study area. (As there are no industrial sources in the town, the $\mathrm{NO}_{2}$ in Guildford has been shown to be mainly due to traffic [14], [15]). It is acknowledged however that direct comparison of the concentration patterns based on the matrix and the tubes respectively is 
not possible, as $\mathrm{NO}_{2}$ tubes measure concentrations of a secondary pollutant while the matrices were based on a primary pollutant and did not account for chemistry effects.

The three models produce in most cases similar spatial distributions and "hot spots". All models show that the areas most vulnerable to air pollution are within the town centre of Guildford, where some street canyon effects are present. The fact that this part of the town is vulnerable to air pollution is also supported by the $\mathrm{NO}_{2}$ tube results which are the highest amongst those available for the wider area of Guildford (Figure 5). Other high vulnerability zones are adjacent to the major trunk road A3, close to the A232 and to a lesser extent the A246. Vulnerable areas are also shown in the proximity of the motorway M25 and the A3, at the North-East of the Borough (in some of these roads there are also some street canyon effects). Some differences however in the exact location and extent of the hot spot zones can be seen. For instance, although both the interaction matrix and ADMS-Urban results show pollution hot spots in the town centre, the highest vulnerability zones according to the interaction matrix models are confined within the street canyons while those from ADMSUrban are shown to spread over greater distances throughout the town centre, and especially towards the A3. Moreover, there are some discrepancies concerning the extent and exact location of the hot spots around the M25/A3 junction area (ADMS-Urban shows a hot spot exactly on the intersection, whereas both matrices show the highest points to be confined inside street canyons along the adjacent roads or in localised spots further from the intersection). Such differences were expected, as direct comparison of the matrix and ADMSUrban results is not possible: the former two are qualitative vulnerability maps, showing how sensitive the system is in variations of a number of factors. The latter focuses on pollutant dispersion rather than on system analysis. It is based on absolute quantitative values of pollutant concentrations, calculated on a very fine grid with a resolution which is twice that of the vulnerability maps.

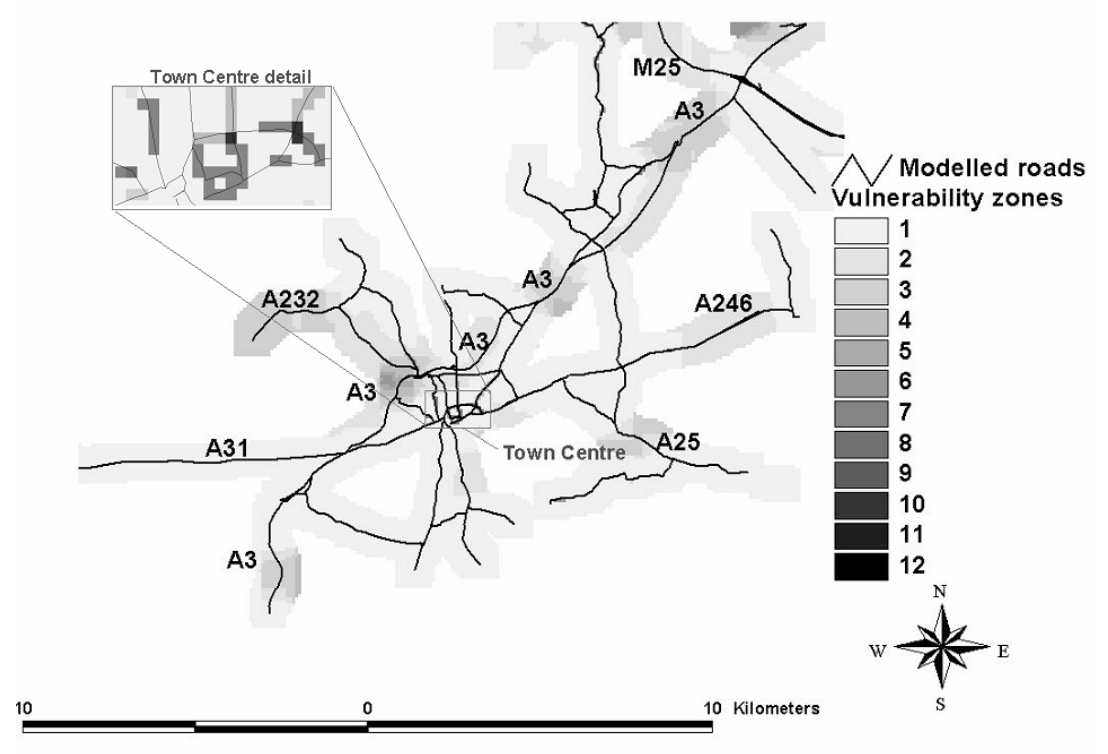

Figure 3. GIS vulnerability map for Guildford town area (constant matrix coefficients)

The effect of using varying matrix coefficients can be clearly seen in the town centre area example: when constant coefficients are used, all street canyons in the town centre are shown to have a relatively high impact, whereas when varying coefficients are used the impact is only pronounced for critical values of $\mathrm{W} / \mathrm{H}$ ratio (where $\mathrm{W}$ and $\mathrm{H}$ are the height and width of the street canyon respectively). The varying coefficient matrix also gives better results in some sections along and adjacent to roads with high emissions (e.g. M25): according to the constant coefficient matrix these are shown to have lower vulnerability than points further away from the source, where more than one areas around roads intersect. This 
is due to the fact that the constant coefficient matrix has not accounted for the reduced effect of the pollutant source as a function of the distance from the source.

Overall, the varying coefficient matrix provides improved results, which are in closer agreement with ADMS-Urban. A further advantage of a varying coefficient coding is that, although it increases the complexity of the tool, it also increases its flexibility and portability to other areas of similar characteristics. It is however important to recognise that the empirical values of the constant coefficient matrix can also be easily adjusted to match other situations.

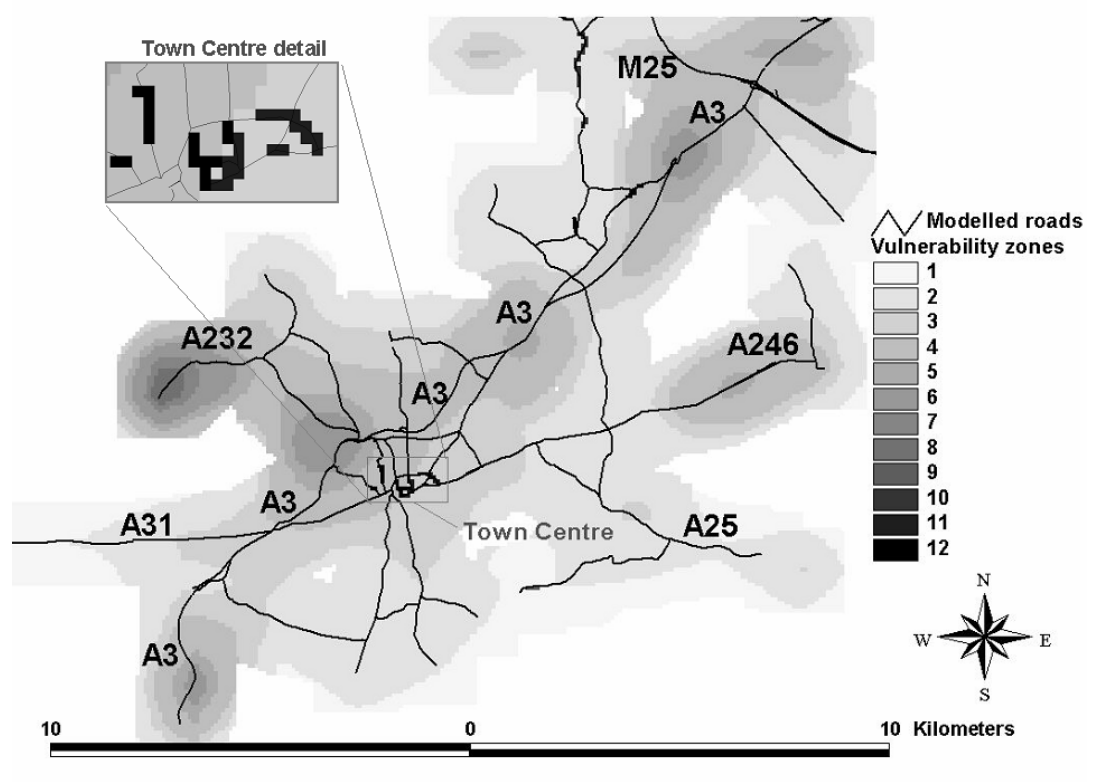

Figure 4. GIS vulnerability map for Guildford town area (varying matrix coefficients)

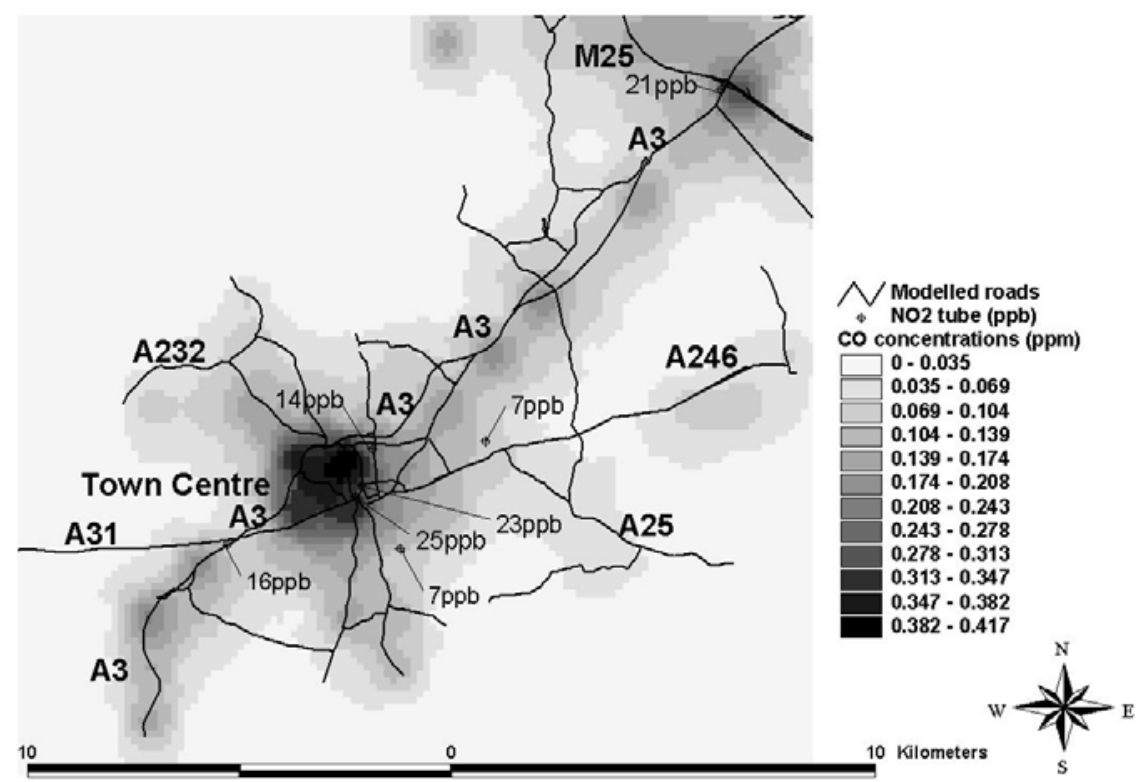

Figure 5. Pollutant concentrations based on ADMS-Urban

Further refinements in the coding could be made, in order to improve predictions. For instance, improved expressions may be introduced to quantify interactions between the variables of the system and the quantification of the matrix interactions could be combined with the probability that each level of interaction occurs. The size of the matrix could increase to incorporate more system variables, including variables of a different nature (e.g. socioeconomic). In the present study the number of variables was purposefully kept small in order 
to verify the results against algorithmic rules and prove the feasibility of the methodology for air quality studies. However the main interest in using this methodology is to solve complex, multidisciplinary problems, involving the interaction of a large number of input variables. Indeed, one of the advantages of the methodology, over a conventional dispersion model (the latter focusing only on pollutant dispersion phenomena) is that it can combine expert information from different fields. Special attention would be required however if different sources/models are used to quantify the interactions. In this case the assumptions should be consistent for all interactions between system variables considered in the matrix.

Although there is scope for further enhancements to the methodology, for a qualitative tool, it is essential to maintain the appropriate balance between such refinements and the resulting increase in complexity. An increase in complexity would also mean that the verification of the matrix results would be more difficult to perform. In such cases other methods such as statistical investigation may be required to check the appropriateness of the matrix.

It is important to note that although extensive parametric studies were performed to quantify the interactions between variables and obtain the weighting factors for the GIS, the user of the finalised tool does not need to repeat this procedure: only the final weightings are imported into the GIS. Hence once the weightings are obtained, the vulnerability analysis is quick and does not require specialised staff. The tool is highly visual and therefore ideal for use by local authorities and urban and transport planners, at preliminary investigation stages.

\section{CONCLUSIONS}

A qualitative tool was developed to study traffic-induced air pollution. Two different interaction-matrix coding techniques were implemented and assessed comparatively. The results from both approaches in terms of vulnerability maps demonstrated that it is feasible to perform preliminary air quality assessments using the proposed methodology. This should reduce the cost of air quality studies for local authorities. Another advantage of this methodology over conventional numerical modelling is that it can provide an integrated assessment of multidisciplinary urban planning problems. In the present research, the method was coded to model the current situation in Surrey. However, the method is believed to be portable, and adaptable to areas of similar characteristics.

\section{REFERENCES}

1. Department of the Environment (1997) The United Kingdom National Air Quality Strategy, The Stationary Office, London

2. Hudson J.A., (1992) Rock Engineering Systems: Theory and Practice, London, Ellis Horwood.

3. Leopold L.B., Clarke F.E., Hanshaw B.B and Balsley J.R., (1971) A procedure for evaluating environmental impact, US Geological Survey (USGS) Circular 645, Government Printing Office, Washington, DC, USGS.

4. Mavroulidou M., Hellawell E.E., Hughes S.J., (2004) A Qualitative Tool Combining an Interaction Matrix and a GIS, to Map Vulnerability to Traffic Induced Air Pollution, Journal of Environmental Management, 70, 283-289.

5. Mavroulidou M., Hughes S.J. and Hellawell E.E., (2006) Developing the interaction matrix technique as a tool assessing the impact of traffic on air quality, Journal of Environmental Management (in press - corrected proof available on line from: www.elsevier.com/locate/jenvman, accessed 27/09/2006).

6. Briggs G.A., (1973) Diffusion estimation for small emissions, ADTL Contribution file No. 79, Atmospheric Turbulence and Diffusion Laboratory, NOAA, Oak Ridge, Tennessee, USA.

7. Hanna S.R., Briggs G.A. and Hosker R.P., (1982) Handbook on Atmospheric Diffusion, US Department of Energy, Technical Information Center, Oak Ridge, Tennessee, USA.

8. Berkowicz R., Hertel O., Sørensen N.N. and Michelsen J.A., (1997) Modelling Air Pollution from Traffic in Urban Areas. In: Flow and Dispersion through Groups of Obstacles, Perkins R.J. and Belcher S.E. (Eds), Clarendon Press. Oxford.

9. Irwin J.S., (1979) A theoretical variation of the wind profile power law exponent as a function of surface roughness and stability, Atmospheric Environment, 13, 191-194. 
10. Clarke R.H., (1979) A Model for Short and Medium Range Dispersion of Radionuclides Released to the Atmosphere, The $1^{\text {st }}$ Report of a Working Group on Atmospheric Dispersion, NRPB-R91, National Radiological Protection Board, Harwell, Didcot, UK

11. Surrey County Council Environment (2000) Local Transport Plan 2001/02 to 2005/06, Surrey County Council, Surrey

12. Engineering Consultancy Division, Scott, Wilson, Kirkpatrick (1996) Surrey County Transportation Model (CTM95), Surrey County Council, Surrey.

13. Department of the Environment, Transport and the Regions (DETR) (1999) Design Manual for Roads and Bridges, Vol 11, Section 3, Part 1: Air Quality, HMSO, London.

14. Cowan I.M., Hellawell E.E., Hughes S.J., (2001) The relationship between traffic throughput and the associated primary pollutants in Surrey, Proceedings of Air Pollution IX Conference, Ancona, Italy, pp. 431-438.

15. Lythe M.S., Hughes S.J. and Hellawell E.E., (2001) Long-term countywide $\mathrm{NO}_{2}$ variations in Surrey, Proceedings of Air Pollution IX Conference, Ancona, Italy, pp. 559-568. 